\title{
Optimal Power Flow Based Architecture Design for Electrical Power System in More-Electric Aircraft
}

\author{
Xin Wang ${ }^{(1)}$, Jason Atkin ${ }^{(2)}$, Serhiy Bozhko ${ }^{(1)}$, Member, IEEE, Christopher Ian Hill ${ }^{(1)}$, Member, IEEE \\ ${ }^{(1)}$ The Department of Electrical and Electronic Engineering \\ ${ }^{(2)}$ School of Computer Science \\ University Of Nottingham \\ Nottingham, United Kingdom \\ xin.wang2@nottingham.ac.uk
}

\begin{abstract}
When designing an electric power system (EPS) architecture for a more electric aircraft (MEA), the total weight of the system is treated as one of the most important criteria. For the weight saving purpose, this paper proposes an optimal power flow (OPF) based architecture design method to minimise the redundancy of the EPS of MEA, which includes reducing the generator overloading requirements for lighter generator design, and cutting down the excessive harness and number of contactors to lighten the wiring system. Based on the formulized mixed-integer linear programming (MILP) model, the addressed optimal architecture design (OAD) problem can be solved by optimising the power routing with minimum transmission losses in different flight stages simultaneously to minimise generator sizing and number of connections. The model contains both decision variables indicating the system architecture (e.g. cables existences and generator capacity) and the ones indicating the power flows with regards to the flight stages. In addition, the total transmission losses in the system are considered by formulizing the non-linear transmission losses of the DC/DC converters and cables losses in model constraints. By minimising the transmission losses, the generator overload capacity, as well as the cable needed for the connections, an optimised architecture with less redundancy and having optimal power routing in different flight stages can be realized, leading to lighter generator and wiring system for EPS.
\end{abstract}

Keywords - optimal architecture design, optimal power flow, transmission losses, wiring redundancy, electric power system, more electric aircraft

\section{INTRODUCTION}

More electric aircraft (MEA) technology is proposed to use more efficient electric energy to replace the pneumatic, hydraulic and mechanical energies for the secondary energy system in conventional aircrafts [1]. This incremental electrification trend is thought to reduce the overall weight of the aircraft, which translates into lower fuel consumption in combination with cost efficiency [2],[3]. Saving one kilogram of weight will reduce costs by approximately $\$ 4500$ for a short/mid-range aircraft over a 20-year-period of operation [4]. In addition, decreasing the consumption of fossil fuels also helps to cut waste gas emissions, which are harmful to the environment [5]. According to the report from the Advisory Council for Aeronautics Research in Europe (ACARE), $\mathrm{CO}_{2}$ emission from aircrafts already account for about $3 \%$ of total global $\mathrm{CO}_{2}$ emission and about $12 \%$ of the $\mathrm{CO}_{2}$ emission of all transportation sources [6].

Since weight saving has significant impacts on aircraft performance and fuel efficiency, optimal architecture design (OAD) targeted on reducing total weight for future MEA becomes a crucial problem for electric power system (EPS) design. This paper aims to propose an OAD method to minimise the redundancy of the EPS in MEA, and therefore reduce the total weight. On the one hand, the method reduces the generator overloading requirements for lighter generator design, and on the other hand, the method cuts down on the excessive cable connections to lighten the wiring system. The optimised architecture must meet the power routing requirement, such as power balance constraints and safe operation constraints, for all flight stages to make sure that the loads can be supplied with needed power from the generators. The optimal power flow (OPF) method is consequently introduced because it is commonly used to route the power with minimum transmission losses or the operation costs in the power system regarding to the load requirements [7],[8],[9]. Typically, this method solves the power scheduling problem and generator sizing problem sequentially regarding to the load requirements at different flight stages [10],[11].

In this paper, an OPF based architecture design method is proposed to solve the optimal power routing in different flight stages simultaneously rather than sequentially. This can be possible since the OAD not only solves the typical power scheduling problem, but also considers an essential constraint that the optimal architecture for different flight stages should remain the same. Hence, in the model formulation of the OAD problem, both variables having changeable values and those remaining unchanged in different flight stages are introduced. The former ones indicate the power flows in different cables/directions and the connection status (on/off) for the contactors, while the latter ones indicate the cable existences and the generator capacity. In addition, when formulizing the OPF model for different flight stages, the non-linear transmission losses for the DC/DC converters and power losses in the cables are considered in constraints to take the overall system transmission losses into account. Therefore, by solving the OPF in different flight stages simultaneously, the proposed OAD method can reduce the transmission losses, the generator overload requirements, as well as the cable needed for the connections in one model, such that an optimised architecture with less weight and having optimal power routing in different flight stages can be realized.

From the literature, several researchers can be observed to have investigated different aspects of the OAD problems. For example, in [1],[5],[12], the authors initially selected several possible EPS architectures by using weight-saving criterion, and then through evaluation of the stability features of the candidates, the optimal EPS architecture is defined. The main focus of [3]-[4] is to assess the advantages of HVDC topologies with AC system, and it is concluded that the converters can be much lighter when using a DC distribution. In [13], the OAD mainly focuses on the optimal load allocation problem for the MEA, because the allocation of electric systems on the busbars is considered to influence the aircraft mass directly. The researchers used four stochastic optimisation methods to assess all possible allocations and proposed the most appropriate algorithm for this problem. In addition, reliability is also an importance 
index when selecting the optimal architecture, for instance, in [14], the potential EPS configurations are evaluated by the safe reliability to provide an optimal solution. However, few of these studies have analysed the optimal EPS architecture supporting the power routing requirements in differently flight stages.

This paper is organized as follow. A conceptual architecture design for a novel HVDC EPS with modular power converters (MPC) is described in Section II, and in Section III, an optimisation model for OAD is formulized by mixed-integer linear programming (MILP) based on the aforementioned conceptual architecture, including the presentation of constraints, objectives and the linearization of the non-linear equations. Section IV illustrates the optimal architectures and the OPF results based on this architecture in different flight stages, and conclusions are drawn in Section V.

\section{Conceptual Design of An EPS ARChitecture}

One conceptual architecture design for a novel HVDC EPS with modular power converters (MPC) is illustrated in Fig. 1, which contains two generators controlled by active AC/DC rectifiers suppling the corresponding $270 \mathrm{~V}$ HV buses [15]. The HV buses provide power to HV loads such as flight control system and de-icing system, and in addition, they provide power to $4 \mathrm{LVDC}$ buses $(28 \mathrm{~V})$ through multicellular ( 8 bidirectional cells) DC/DC converter. DC/DC converters are bidirectional to ensure the availability for transferring power from storage devices to the HV side, which will be further discussed in our following papers. In Fig. 1, HVDC LOADS $1 \& 2$ represent the HV loads connected to HV bus1\&2, while the LVDC LOADS1/2/3/4 represent the LV loads connected to LV bus $1 / 2 / 3 / 4$.

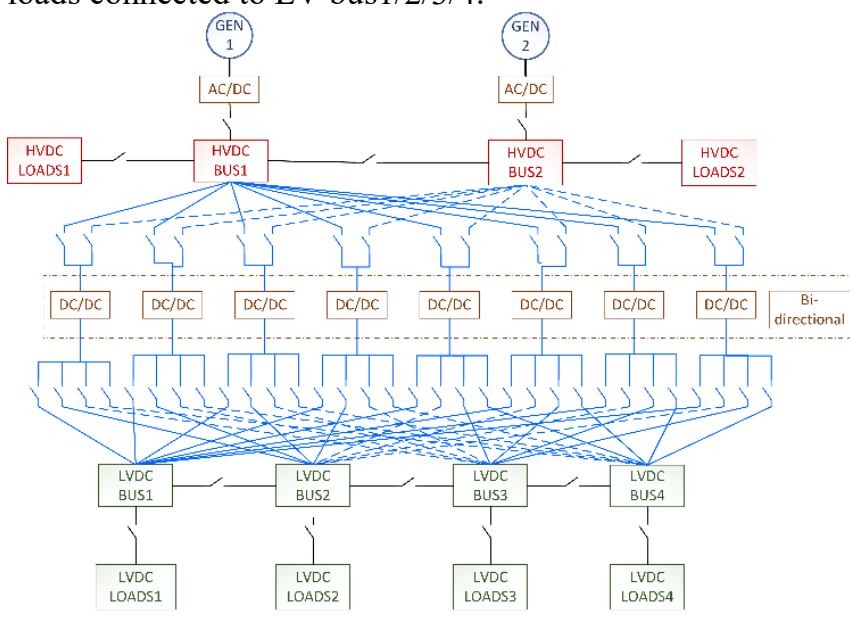

Fig. 1 Conceptual architecture design of MPC HVDC EPS

In this conceptual architecture, the connection between the generators, $\mathrm{HV} / \mathrm{LV}$ buses, and $\mathrm{HV} / \mathrm{LV}$ loads are similar to the conventional architecture of an EPS in aircraft [5]. However, the MPC HVDC EPS introduces multiple DC/DC conversions, which offers the system a wide range of connection possibilities. This has huge flexibility benefits, but including all of the connections in the system would result in an excessive increase in the total weight of the system since not all of the connections will be needed in each flight stage and some may not be used in any of the flight stages. Thus, an optimisation method is required to minimise this connection redundancy for weight reduction, but the optimised architecture should meet the requirement for power transmission from the power sources to the changing loads in each of the different flight stages, ensuring that power flows are still optimised.

In addition to connection redundancy, system sizing is also considered as another aspect of the architecture design problem. Minimising the generator overload redundancy to have a lighter generator can also contributes to the weight reduction for the aircraft.

\section{OPtIMISATION MODEL FOR ARCHITECTURE DESIGN}

Minimising the connection redundancy and system sizing to reduce the total weight of the EPS are the two main OAD issues focused on in this paper. To guarantee the power routing in different flight stages, the OPF model is adopted in the architecture design, because it can ensure that the power is routed with minimal transmission losses in the power system considering the load requirements based on system operation constraints. If each stage is solved independently, it is quite likely that different routes (and connections) for power flow will be chosen in different stages, even when it may be as efficient (or almost as efficient) to utilise the same connections (potentially in different ways) across stages. This is important for weight reduction since a connection is needed even if it is only utilised in a few, or one, of the flight stage. Therefore, an adapted OAD model is formulized in this section to solve the OPF for different flight stages simultaneously, introducing a cost for any connection which is needed (thus reducing the number of connections used and encouraging reuse between stages) while also optimising power flow within each stage.

\section{A. Notations} I.

The main symbols used in this paper are shown in TABLE

TABLE I. Notations for Optimal ARChitecture Design Problem FORMULATION

\begin{tabular}{|c|c|}
\hline \multicolumn{2}{|r|}{ Indices and numbers } \\
\hline$S$ & Index for flight stages \\
\hline$j, k$ & Index for HV and LV buses respectively \\
\hline$c$ & Index for DC/DC converter \\
\hline$p, q$ & Index for HV and LV loads respectively \\
\hline \multicolumn{2}{|r|}{ Continuous variables } \\
\hline$P^{G c a p}$ & Generator power capacity \\
\hline$P_{s j}^{G}$ & $\begin{array}{l}\text { Magnitude of the power flow from generator to } \\
\text { corresponding HV bus } j \text { at stage } s\end{array}$ \\
\hline$P_{s j c}^{H V C}$ & $\begin{array}{c}\text { Power flowing from } \mathrm{HV} \text { bus } j \text { to } \mathrm{DC} / \mathrm{DC} \\
\text { converter } c \text { at stage } s\end{array}$ \\
\hline$P_{s j c}^{\prime H V C}$ & $\begin{array}{c}\text { Power flowing from DC/DC converter } c \text { to HV } \\
\text { bus } j \text { at stage } s\end{array}$ \\
\hline$P_{S C k}^{C L V}$ & $\begin{array}{c}\text { Power flowing from DC/DC converter } c \text { to } \mathrm{LV} \\
\text { bus } k \text { at stage } s\end{array}$ \\
\hline$P_{\text {sck }}^{\prime C L V ~}$ & $\begin{array}{l}\text { Power flowing from LV bus } k \text { to DC/DC } \\
\text { converter } c \text { at stage } s\end{array}$ \\
\hline$P_{s p}^{H V L}$ & $\begin{array}{l}\text { Power drawn from HV bus by HV load } p \text { at } \\
\text { stage } s\end{array}$ \\
\hline$P_{s q}^{L V L}$ & $\begin{array}{l}\text { Power drawn from LV bus by LV load } q \text { at } \\
\text { stage } s\end{array}$ \\
\hline$P_{s j j^{\prime}}^{H V}$ & $\begin{array}{c}\text { Power flowing from HV bus } j \text { to HV bus } j^{\prime} \text { at } \\
\text { stage } s\end{array}$ \\
\hline$P_{s k k \prime}^{L V}$ & Power flowing from LV bus $k$ to LV bus $k^{\prime}$ at \\
\hline
\end{tabular}


stage $S$

\begin{tabular}{|c|c|}
\hline & stage $S$ \\
\hline \multicolumn{2}{|r|}{ Binary variables } \\
\hline$E_{j c}^{H V C}$ & $\begin{array}{l}\text { Existence of the connection between HV bus } j \\
\text { and converter } c^{(1)}\end{array}$ \\
\hline$E_{c k}^{C L V}$ & $\begin{array}{c}\text { Existence of the connection between LV bus } k \\
\text { and DC/DC converter } c^{(1)}\end{array}$ \\
\hline$\beta_{s j c}$ & $\begin{array}{l}\text { Connection status between HV bus } j \text { and } \\
\text { DC/DC converter } c \text { at stage } s\end{array}$ \\
\hline$f_{s j c}$ & $\begin{array}{l}\text { Indicator for power flow direction from HV } \\
\text { bus } j \text { to } \mathrm{DC} / \mathrm{DC} \text { converter } c \text { at stage } S^{(3)}\end{array}$ \\
\hline$f_{s j c}^{\prime}$ & $\begin{array}{l}\text { Indicator for power flow direction from LV bus } \\
k \text { to DC/DC converter } c \text { at stage } s^{(3)}\end{array}$ \\
\hline$\theta_{s c k}$ & $\begin{array}{c}\text { Connection between LV bus } k \text { and cell } c \text { at } \\
\text { stage } s^{(2)}\end{array}$ \\
\hline$f_{s c k}$ & $\begin{array}{c}\text { Indicator for power flow direction from } \\
\text { DC/DC converter } c \text { to LV bus } k \text { at stage } s^{(3)}\end{array}$ \\
\hline$f_{s c k}$ & $\begin{array}{c}\text { Indicator for power flow direction from } \\
\text { DC/DC converter } c \text { to LV bus } k \text { at stage } s^{(3)}\end{array}$ \\
\hline$f_{s c k}^{\prime}$ & $\begin{array}{l}\text { Indicator for power flow direction from LV bus } \\
k \text { to DC/DC converter } c \text { at stage } s^{(3)}\end{array}$ \\
\hline$x_{s j j^{\prime}}$ & $\begin{array}{c}\text { Connection status between HV bus } j \text { and HV } \\
\text { bus } j^{\prime} \text { at stage } S^{(2)}\end{array}$ \\
\hline$f_{s j j^{\prime}}$ & $\begin{array}{l}\text { Indicator for power flow direction from HV } \\
\text { bus } j \text { to HV bus } j^{\prime} \text { at stage } s^{(3)}\end{array}$ \\
\hline$y_{s k k^{\prime}}$ & $\begin{array}{l}\text { Connection statues between LV bus } k \text { and LV } \\
\text { bus } k^{\prime} \text { at stage } s^{(2)}\end{array}$ \\
\hline$f_{s k k^{\prime}}^{H V}$ & $\begin{array}{l}\text { Indicator for power flow direction from LV bus } \\
k \text { to LV bus } k^{\prime} \text { at stage } s^{(3)}\end{array}$ \\
\hline \multicolumn{2}{|r|}{ Parameters } \\
\hline$\eta_{s c}, \eta_{s c}^{\prime}$ & $\begin{array}{l}\text { Efficiency of DC/DC converter in buck/boost } \\
\text { mode at stage } s\end{array}$ \\
\hline$\epsilon_{j c}^{H V C}$ & $\begin{array}{c}\text { Transmission efficiency in cables between the } \\
\text { HV bus } j \text { and DC/DC converter } c\end{array}$ \\
\hline$\epsilon_{c k}^{C L V}$ & $\begin{array}{l}\text { Transmission efficiency in cables between the } \\
\text { DC/DC converter } c \text { and LV bus } k\end{array}$ \\
\hline$w_{j c}^{H V C}$ & $\begin{array}{l}\text { Penalty of cable weight between the HV bus } j \\
\text { and DC/DC converter } c\end{array}$ \\
\hline$w_{c k}^{C L V}$ & $\begin{array}{c}\text { Penalty of cable weight between the DC/DC } \\
\text { converter } c \text { and LV bus } k\end{array}$ \\
\hline
\end{tabular}

(1) These binary variables take the value 1 if the connection exists, and 0 otherwise.

(2) These binary variables take the value 1 if the connection is being used in the specified stage.

(3) A value of 1 means that there can be a non-negative power flow in that stage from the first named component to the second, whereas a value of 0 indicates no power flow is possible in that direction (i.e. the magnitude of the power flow must be zero).

\section{B. Objective function}

The proposed OAD problem aims to minimise the connection redundancy between the HV/LV buses and the converters, as well as minimise the generator capacity and total power needed from the generator in all flight stages. This multi-objective function can be presented as (1)-(4).

$$
\begin{gathered}
O(X)=w_{1} f_{c a b}+w_{2} f_{\text {Pcap }}+w_{3} f_{P_{\text {tot }}} \\
f_{c a b}=\sum_{j} \sum_{c} w_{j c}^{H V C} E_{j c}^{H V C}+\sum_{c} \sum_{k} w_{c k}^{C L V} E_{c k}^{C L V}
\end{gathered}
$$

$$
\begin{gathered}
f_{\text {Pcap }}=P^{G \text { cap }} \\
f_{P_{\text {tot }}}=\sum_{s} \sum_{j} P_{s j}^{G}
\end{gathered}
$$

where $X$ is the set of decision variables, $f_{c a b}$ is the cost function for the existence of the connections, $f_{\text {Pcap }}$ is the cost function for the generator capacity, $f_{P_{t o t}}$ is the cost function for the total power supplied from the generators in all flight stages, which also indicates minimising the total transmission losses, and $w_{1}, w_{2}, w_{3}$ are the weights of each cost function, allowing the user to balance priorities.

\section{Constraints}

1) Connection existance constraints: The connection status between HV/LV buses and DC/DC converters can be set for a flight stage only when the cables and contactors exist for this connection, i.e.,

$\forall s \in \mathbb{S}, \forall j \in \mathbb{J}, \forall c \in \mathbb{C}, \forall k \in \mathbb{K}$,

$$
\begin{aligned}
& 0 \leq \beta_{s j c} \leq E_{j c}^{H V C} \\
& 0 \leq \theta_{s c k} \leq E_{c k}^{C L V}
\end{aligned}
$$

where $\mathbb{S}$ is the set of flight stages, $\mathbb{J}$ is the set of HV buses, $\mathbb{C}$ is the set of DC/DC converters, and $\mathbb{K}$ is the set of LV buses.

2) Power balance constraints with consideration of power efficiency and bidirectional power flow: The sum of powers flowing into/out of each bus equals zero, while the power flowing out of each converter is less than the power flowing into it because of the converter efficiency. In addition, the novel HVDC MPC EPS introduces bidirectional power flow by operating the converters in either buck or boost mode, thus a non-negative decision variable is adopted to represent the power flow in each direction.

The power balance equations for HV/LV buses with the consideration of the power losses during the conversion are presented in (7) and (8). For each HV bus at each flight stage, the input power include power from generator, the adjacent HV buses, and the boost mode converters, and the output power consists of those flowing into HV loads, the adjacent $\mathrm{HV}$ buses and the converters in buck mode. $\epsilon_{j c}^{H V C}$ represents the transmission efficiencies between $\mathrm{HV}$ buses and converters, which is assumed constant, to indicate the cable losses. The LV buses have the similar power balance constraints indicating that the net power from converters and adjacent LV buses supplies LV loads at each flight stage. $\forall s \in \mathbb{S}, \forall j \in \mathbb{J}, \forall c \in \mathbb{C}, \forall k \in \mathbb{K}$,

$$
\begin{gathered}
P_{s j}^{G}+\sum_{c}\left(\epsilon_{j c}^{H V C} P_{s j c}^{\prime^{H V C}}-P_{s j c}^{H V C}\right)-\sum_{p} P_{s p}^{H V L} \\
-\sum_{j \neq j^{\prime}}\left(P_{s j j^{\prime}}^{H V}-P_{s j^{\prime} j}^{H V}\right)=0 \\
\sum_{c}\left(\epsilon_{c k}^{C L V} P_{s c k}^{C L V}-P^{\prime C L V}{ }_{s c k}\right)-\sum_{s q} P_{s q}^{L V L} \\
-\sum_{k \neq k^{\prime}}\left(P_{s k k^{\prime}}^{L V}-P_{s k^{\prime} k}^{L V}\right)=0
\end{gathered}
$$

The converters operate in buck mode to transfer power from the HV side to LV side, while the power flows in the opposite direction when they are in boost mode. Considering the nonlinear converter efficiency and the constant transmission efficiency of the cable, (9) represents the power balance for the converters in each flight stage.

$$
\begin{gathered}
\left(\eta_{s c} \sum_{j}\left(\epsilon_{j c}^{H V C} P_{s j c}^{H V C}\right)-\sum_{j} P_{s c j}^{H V C}\right) \\
-\left(\sum_{k} P_{s c k}^{L V C}-\eta_{s c}^{\prime} \sum_{k}\left(\epsilon_{c k}^{C L V} P_{s c k}^{\prime L V C}\right)\right)=0
\end{gathered}
$$

3) Connection constraints: In each flight stage, the contactors are controlled to change the connection status of 
the buses and converters to provide power paths and prevent unexpected connection conditions. For instance, each DC/DC converter can be connected to no more than one $\mathrm{HV}$ and LV bus as presented in (10). In addition, in the aircraft EPS, the $\mathrm{HV} / \mathrm{LV}$ buses can have interconnections only when having generator failures and the system operates in fault mode as shown in (11), where $\alpha_{s j}^{G}$ is the generator connection status, and $N^{G}$ is the number of the generators.

$$
\begin{gathered}
0 \leq \sum_{j} \beta_{s j c} \leq 1,0 \leq \sum_{k} \theta_{s c k} \leq 1 \\
x_{s j j^{\prime}}+\sum_{j} \alpha_{s j}^{G} \leq N^{G}, y_{s k k^{\prime}}+\sum_{j} \alpha_{s j}^{G} \leq N^{G}
\end{gathered}
$$

4) Unidirectional constraints: The power cannot flow in both directions simultaneously despite the connections being bidirectional, because the DC/DC converter can only operate in either buck or boost mode rather than in both mode simultaneously. Similar rules apply for HV/LV bus interconnections. These unidirectional constraints are represented in (12) and (13).

$$
\begin{gathered}
f_{s j c}+f_{s j c}^{\prime} \leq \beta_{s j c}, f_{s c k}+f_{s c k}^{\prime} \leq \theta_{s c k} \\
f_{s j j^{\prime}}^{H V}+f_{s j^{\prime} j}^{H V} \leq x_{s j j^{\prime}}, f_{s k k^{\prime}}^{L V}+f_{s k^{\prime} k}^{L V} \leq y_{s k k^{\prime}}
\end{gathered}
$$

5) Bounds constraints: The power flowing through converters and cables should not exceed their power capacities. In addition, the power flow in each direction is restricted to 0 when either the direction indicators/connection status values 0 . Therefore, the group of power upper and lower bounds can be given as follows

$$
\mathbf{0} \leq \boldsymbol{P} \leq \boldsymbol{F} \times \boldsymbol{P}_{\text {max }}
$$

where $\boldsymbol{P}=\left[P_{s j c}^{H V C}, P_{s c k}^{C L V}, P_{s c k}^{\prime C L V}, P_{s j c}^{\prime H V C}, P_{s j j^{\prime}}^{H V}, P_{s k k^{\prime}}^{L V}\right]^{T}$ is the vector of power flows in each direction, $\boldsymbol{F}=\left[f_{s j c}, f_{s c k}\right.$, $\left.f_{s c k}^{\prime}, f_{s j c}^{\prime}, f_{s j j^{\prime}}^{H V}, f_{s k k^{\prime}}^{L V}\right]^{T}$ is the vector of the direction indicators, in addition, $\boldsymbol{P}_{\max }=\left[P_{\max }^{C}, P_{\max }^{C}, P_{\max }^{C}, P_{\max }^{C}\right.$, $\left.P_{k k^{\prime}{ }_{\text {_max }}}^{L V}, P_{k k^{\prime}{ }_{\text {_max }}}^{L V}\right]^{T}$ is the vector of the power capacities regarding to $\boldsymbol{P}$.

6) Generator capacity constraints: The generator capacity is defined as a decision variable to optimise the maximum power needed from each generator. Generators should operate within this capacity which have a predefined upper bound $P_{\max }^{G}$, i.e.,

$$
0 \leq P_{s j}^{G} \leq P^{G c a p}, 0 \leq P^{G c a p} \leq P_{\max }^{G}
$$

\section{Linearization}

The aforementioned formulation contain nonlinear constraints (9) because the converter efficiency functions $\eta_{s c}$ and $\eta^{\prime}{ }_{s c}$ have nonlinear relationships with regard to the input power, which is demonstrated in Fig. 2 with the blue curve. This relation can be represented by $(16)$, where $P_{\text {sjc_in }}^{H V C}=$ $\epsilon_{j c}^{H V C} P_{s j c}^{H V C}$ and $P_{s c k_{-} i n}^{\prime L V C}=\epsilon_{c k}^{C L V} P_{s c k}^{\prime L V C}$ are introduced to represent the total input power of $\mathrm{DC} / \mathrm{DC}$ converters in buck/boost mode respectively.

$$
\eta_{s c}=f\left(\sum_{j} P_{s j c_{-} i n}^{H V C}\right), \eta_{s c}^{\prime}=f\left(\sum_{k} P_{s c k_{-} i n}^{\prime L V C}\right)
$$

In order to solve this model automatically using a (fast) mixed integer linear programming solver (see Section IV), it is necessary to formulate it as a function which the solver can deal with. This nonlinearity can be linearized using piecewiselinear functions, which can be utilised by solvers (despite potentially introducing some slow-down to the solution method). Instead of formulating the nonlinear efficiency in (9), the output powers of the DC/DC converters after considering the efficiencies are segmented to form a piecewise-linear function, for the output power in buck mode, which can be expressed by Equation (17).

$$
\begin{aligned}
& P_{\text {Sc_out }}^{H V C}=\eta_{c} \sum_{j} P_{\text {sjc_in }}^{H V C} \approx f_{p}\left(\sum_{j} P_{\text {sjc_in }}^{H V C}\right) \\
& =\left\{\begin{array}{c}
k_{1} \sum_{j} P_{s j c_{-} i n}^{H V C}+b_{1}\left(m_{0} \leq \sum_{j} P_{s j c_{-} i n}^{H V C} \leq m_{1}\right) \\
k_{2} \sum_{j} P_{s j c_{-} i n}^{H V C}+b_{2}\left(m_{1} \leq \sum_{j} P_{s j c_{-} i n}^{H V C} \leq m_{2}\right) \\
k_{3} \sum_{j} P_{s j c_{-} i n}^{H V C}+b_{3}\left(m_{2} \leq \sum_{j} P_{\text {sjc_in }}^{H V C} \leq m_{3}\right) \\
\vdots \\
k_{n} \sum_{j} P_{\text {sjc_in }}^{H V C}+b_{n}\left(m_{n-1} \leq \sum_{j} P_{s j c_{-} i n}^{H V C} \leq m_{n}\right)
\end{array}\right.
\end{aligned}
$$

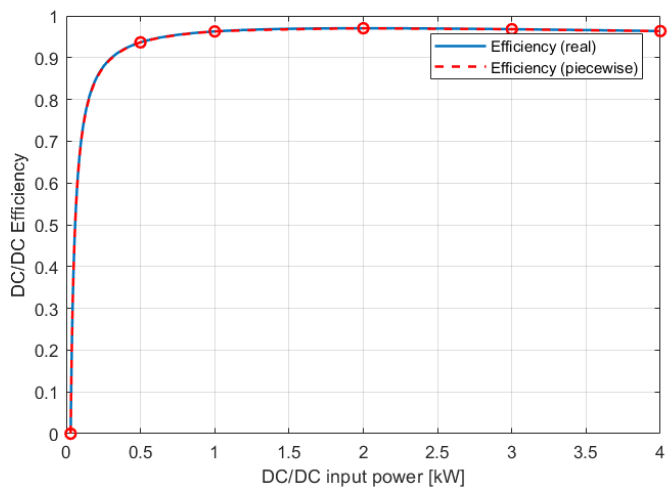

Fig. 2 The piecewise function derived efficiency curve compared to the typical efficiency curve

The approximated converter efficiency can be derived from the piecewise functions of the output power using (18). In Fig. 2, the curve in red shows the approximated converter efficiency from the piecewise functions, compared to the real efficiency curve, showing that the approximation has good accuracy.

$$
\widetilde{\eta_{s c}}=\frac{k_{m} P_{s j c_{i n}}^{H V C}+b_{m}}{P_{\text {sjc_in }}^{H V C}}=k_{m}+\frac{b_{m}}{P_{\text {sjc_in }}^{H V C}}
$$

\section{SimULATION RESULTS}

In this section, the OAD model proposed in Section III is applied into the conceptual architecture of the MPC HVDC EPS in Fig. 1. Since the constraints has been linearized, the model can be formulated as a Mixed-Integer Linear Programming (MILP) model and solved using CPLEX (a MILP solver). The model will deal with eight flight stages simultaneously, including taxiing to the runway, take-off, climbing, cruise, descent, approach, landing, and taxiing to the terminal.

In this architecture optimisation, all the electrical components and generators are assumed to be in normal operating condition. The optimised architecture of the MPC HVDC EPS is illustrated in Fig. 3. The optimisation reduces more than a half of the redundancy of connections between HV/LV buses and the eight DC/DC converters. Fig. 4 presents the power allocation of the generators in eight flight stages with HV/LV loads profiles based on this optimal architecture. The power capacity for each generator is almost $70 \mathrm{~kW}$, and in each flight stage, the generators have to supply the power 
requirements of the loads as well as covering the transmission losses.

To look into how this optimal architecture operates to route power from the generators to the loads with one of the objectives to minimise the transmission losses, the usage conditions of eight DC/DC converter (labelled as C1, C2,..., C8) are illustrated in Fig. 5 and Fig. 6, which show the input power and efficiency of each converter in every stage respectively. The input power and the efficiency for each converter corresponds to the efficiency curve in Fig. 2.

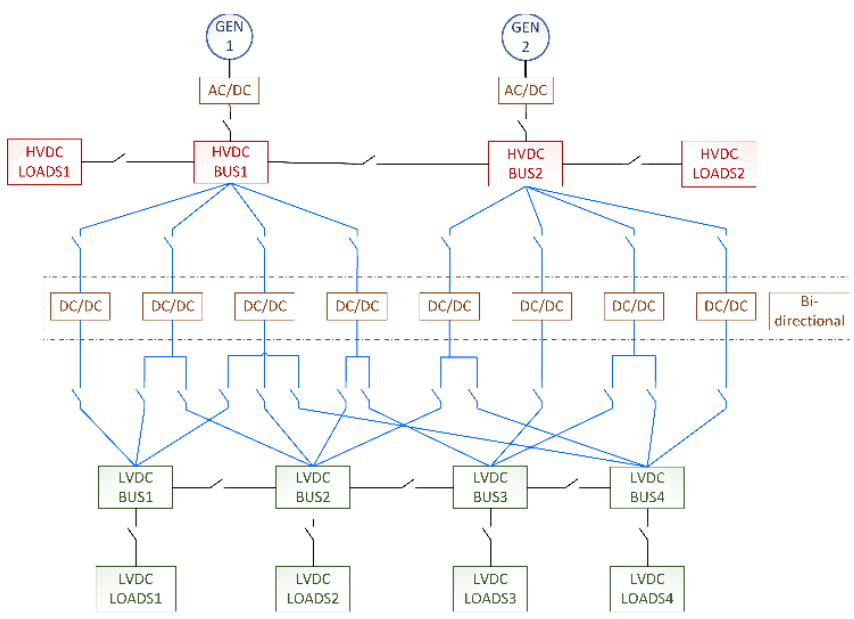

Fig. 3 Optimised architecture design of MPC HVDC EPS
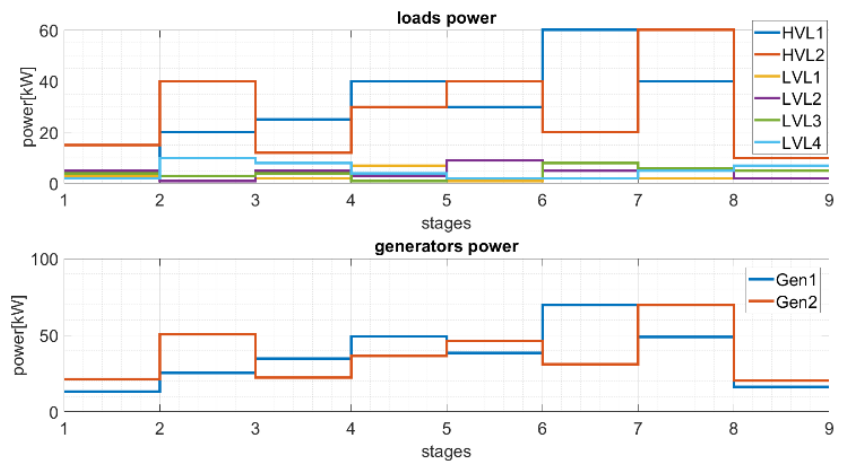

Fig. 4 Load requirements and generator power in different flight stages
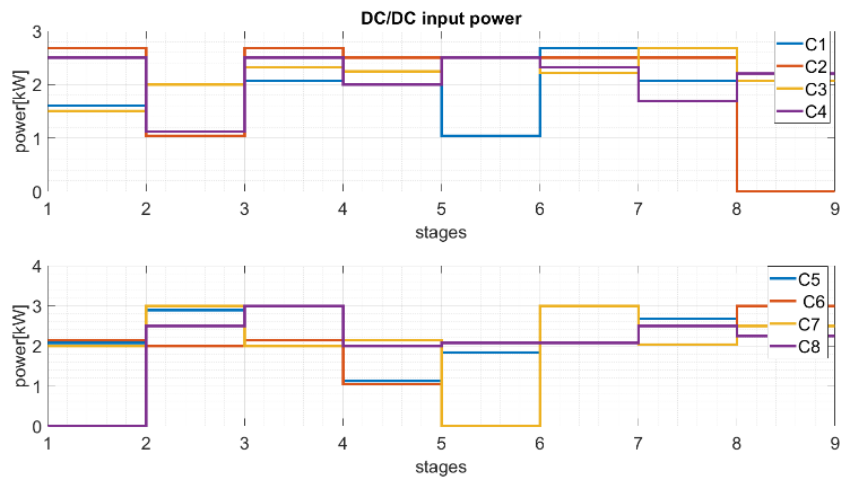

Fig. 5 Input power of eight DC/DC converters in different flight stages
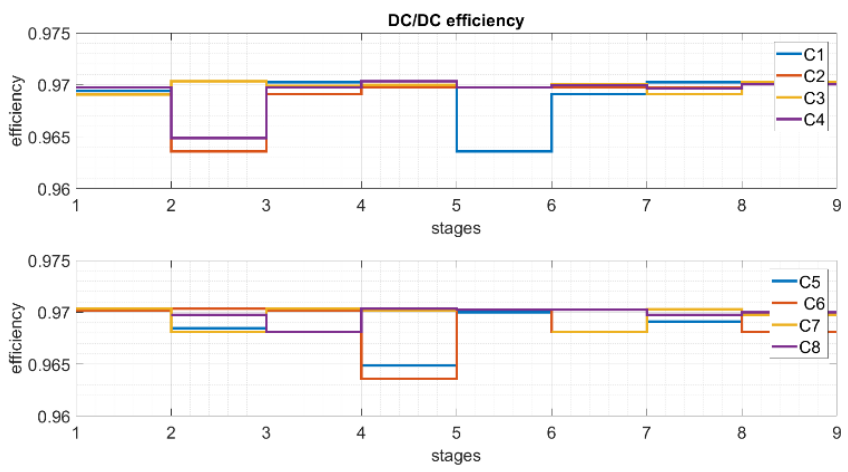

Fig. 6 Efficiency of eight DC/DC converters in different flight stages

In addition, the contactor connection status between the $\mathrm{HV}$ buses and DC/DC converters are presented in Fig. 7 which use label Sh2c11 meaning the connection between HVDC bus 1 and DC/DC1, etc. Fig. 7 shows that all the eight contactors between HV buses and converters in Fig. 3 stay connected in all flight stages. Similarly, the connection status of DC/DC converters and LV buses are illustrated in Fig. 8, where labels are abbreviations of the connections, for example, Sc2111 is used to represent the connection between $\mathrm{DC} / \mathrm{DC} 1$ and LVDC bus1. In contrast to the HV sides, the connections of LV sides keep changing to supply the LV loads.

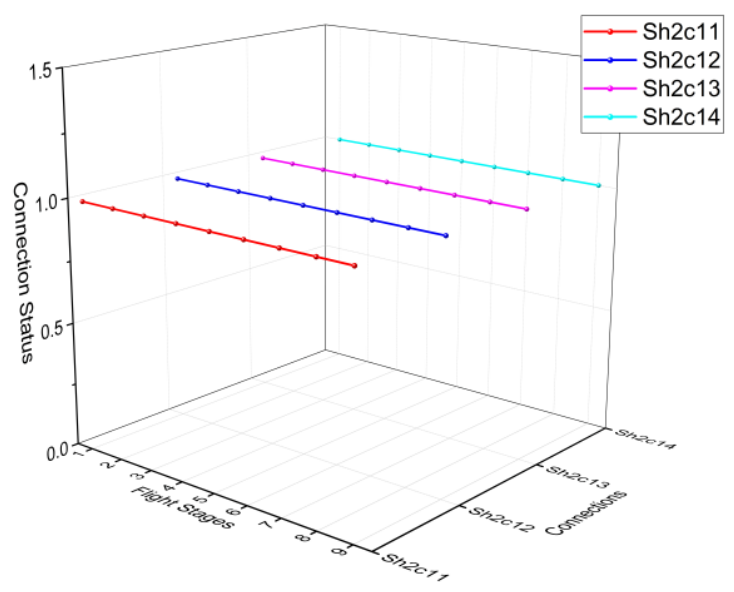

(a)

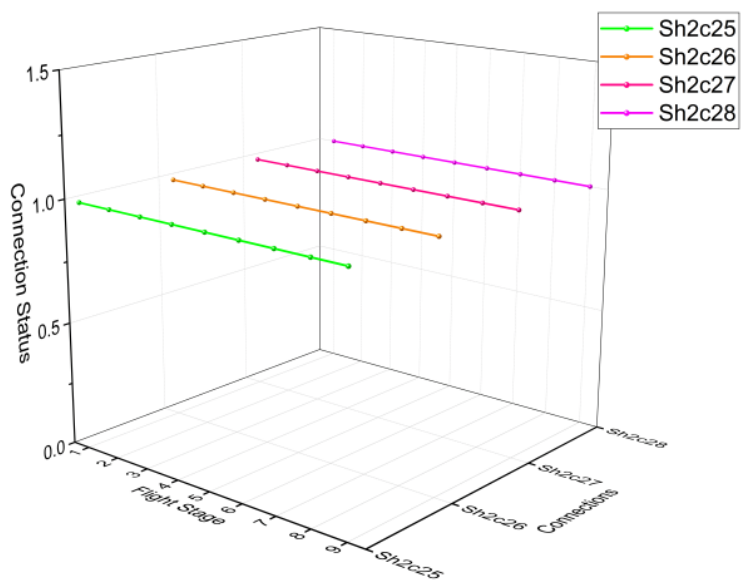

(b)

Fig. 7 Contactor status between HV buses to eight DC/DC converters in different flight stages 


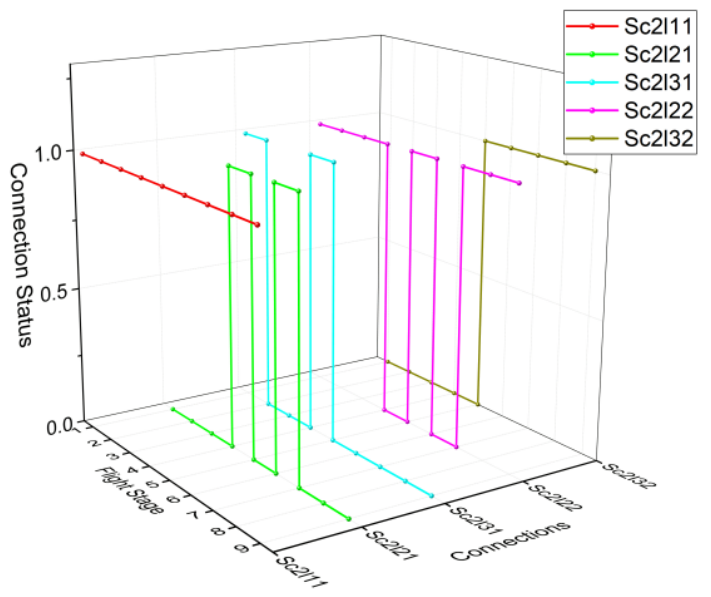

(a)

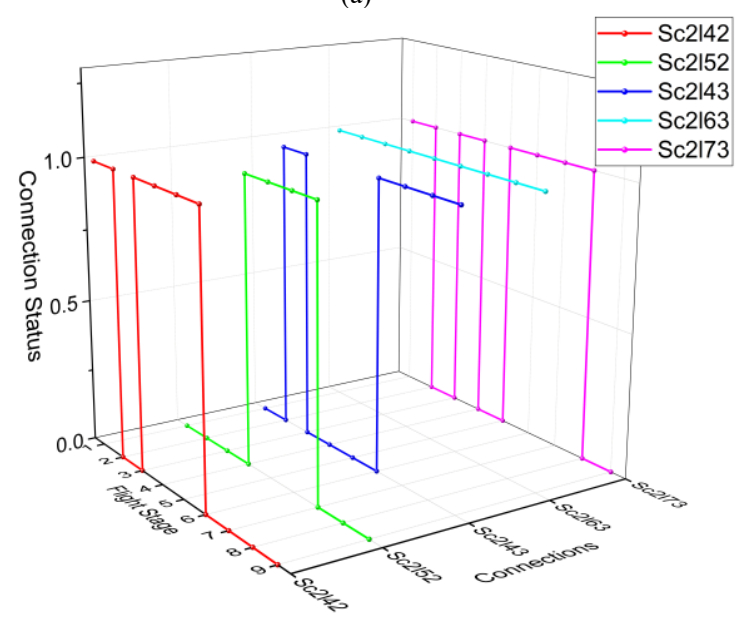

(b)

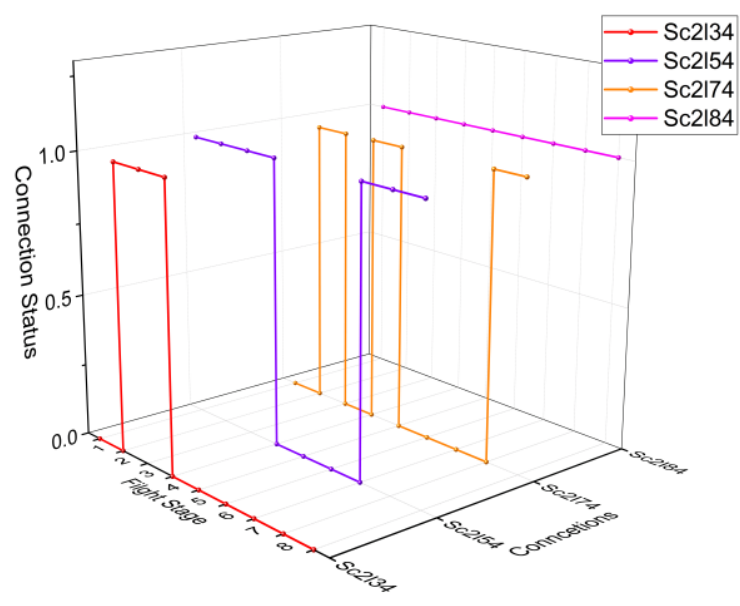

(c)

Fig. 8 Contactor status between eight DC/DC converters and LV buses in different flight stages

\section{CONCLUSIONS}

This paper proposes an OPF based architecture design method to minimise the generator overload requirements and wiring system redundancy of the EPS of MEA aiming to reduce the total weight. A mathematical model based on this method is formulized to optimise the power routing with minimum transmission losses in different flight stages simultaneously. Built with MILP, the model is applied to a conceptual architecture of novel HVDC EPS with modular power converters to reduce the transmission losses in different flight stages, each generator capacity, as well as the number of the cables needed for the connections between the HV and LV buses. From the simulation results, the optimal architecture for the MPC HVDC EPS reduced more than a half of the cables needed for the connection between HV and LV buses, and for each flight stage, the optimal power routing solution based on this optimal architecture is provided. The generator capacity can be also obtained from this power routing solution.

\section{ACKNOWLEDGMENT}

This work is funded by the INNOVATIVE doctoral programme. The INNOVATIVE programme is partially funded by the Marie Curie Initial Training Networks (ITN) action, and partially by the Institute for Aerospace Technology (IAT) at the University of Nottingham.

\section{REFERENCES}

[1] Y. Xu, Z. Zhang, J. Li, and Y. Yan, "Architecture analysis and optimization of high voltage DC parallel electric power system for more electric aircraft," AUS 2016 - 2016 IEEE/CSAA Int. Conf. Aircr. Util. Syst., pp. 244-249, 2016.

[2] T. Kostakis, P. J. Norman, and S. J. Galloway, "Assessing network architectures for the more electric engine and aircraft," Proc. Univ. Power Eng. Conf., 2014.

[3] J. Brombach, B. Nya, M. Johannsen, D. Schulz, and A. O. Gmbh, "Comparison of Different Electrical HVDC- Architectures for Aircraft Application," Electr. Syst. Aircraft, Railw. Sh. Propuls. (ESARS), 2012, pp. 1-6, 2012.

[4] J. Brombach, T. Schröter, and D. Schulz, "Optimizing the Weight of an Aircraft Power Supply System through a + / - 270 VDC Main Voltage," no. 1, pp. 47-50, 2012.

[5] J. Chen, C. Wang, and J. Chen, "Investigation on the Selection of Electric Power System Architecture for Future More Electric Aircraft," IEEE Trans. Transp. Electrif., vol. 7782, no. c, pp. 1-1, 2018.

[6] A. Garcia and B. Bettignies-Thiébaux, "Aeronautics and Air Transport: Beyond Vision 2020 (Towards 2050)," Aeronaut. Air Transp. Beyond Vis., vol. 2020, no. Towards 2050, pp. 1-100, 2010.

[7] M. Aragüés-Peñalba, A. Egea-Àlvarez, O. Gomis-Bellmunt, and A. Sumper, "Optimum voltage control for loss minimization in HVDC multi-terminal transmission systems for large offshore wind farms," Electr. Power Syst. Res., vol. 89, pp. 54-63, 2012.

[8] J. Ma, L. Yuan, Z. Zhao, and F. He, "Transmission Loss OptimizationBased Optimal Power Flow Strategy by Hierarchical Control for DC Microgrids," IEEE Trans. Power Electron., vol. 32, no. 3, pp. 19521963, 2017.

[9] W. Shi, X. Xie, C. C. Chu, and R. Gadh, "Distributed Optimal Energy Management in Microgrids," IEEE Trans. Smart Grid, vol. 6, no. 3, pp. 1137-1146, 2015 .

[10] A. Barzegar, R. Su, C. Wen, L. Rajabpour, Y. Zhang, and M. Y. Lee, "Intelligent Power Allocation and Load Management of More Electric Aircraft," Ieee Peds, no. June, pp. 533-538, 2015.

[11] Y. Zhang, R. Su, C. Wen, M. Y. Lee, and C. Gajanayake, "Distributed power allocation and scheduling for electrical power system in more electric aircraft," IECON 2016 - 42nd Annu. Conf. IEEE Ind. Electron. Soc., pp. 102-107, 2016

[12] J. Chen, C. Wang, and J. Chen, "Investigation on the selection of a more suitable power system architecture for future more electric aircraft from the prospective of system stability," IEEE Int. Symp. Ind. Electron., vol. 0 , pp. 1861-1867, 2017

[13] X. Giraud et al., "Load allocation problem for optimal design of aircraft electrical power system," Int. J. Appl. Electromagn. Mech., vol. 43, no. 1-2, pp. 37-49, 2013.

[14] H. Qi, Y. Fu, X. Qi, and Y. Lang, "Architecture optimization of more electric aircraft actuation system," Chinese J. Aeronaut., vol. 24, no. 4, pp. 506-513, 2011.

[15] S. Bozhko, M. Liserre, K. Al-Haddad, G. Buticchi, and P. Wheeler, "On-board Microgrids for the More Electric Aircraft - Technology Review," IEEE Trans. Ind. Electron., vol. 66, no. 7, pp. 1-1, 2018. 